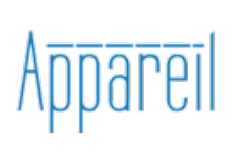

Appareil

$13 \mid 2014$

Trauma et création

\title{
Un coma ça tape où? Incorporer le corps dans le voir
}

\section{Germain Rœsz}

\section{(2) OpenEdition}

1 Journals

Édition électronique

URL : http://journals.openedition.org/appareil/2033

DOI : 10.4000/appareil.2033

ISSN : 2101-0714

Éditeur

MSH Paris Nord

Référence électronique

Germain Rœsz, « Un coma ça tape où ? Incorporer le corps dans le voir », Appareil [En ligne], 13 | 2014, mis en ligne le 23 juillet 2014, consulté le 30 juillet 2020. URL : http://journals.openedition.org/ appareil/2033; DOI : https://doi.org/10.4000/appareil.2033

Ce document a été généré automatiquement le 30 juillet 2020.

\section{(c) (i) (9)}

Appareil est mis à disposition selon les termes de la Licence Creative Commons Attribution - Pas d'Utilisation Commerciale - Pas de Modification 4.0 International. 


\title{
Un coma ça tape où? Incorporer le corps dans le voir
}

\author{
Germain Rœsz
}

1 J'ai écrit ce texte à la demande de Jean-Louis Déotte.

2 Depuis des années nos rencontres se multiplient, nous parlons de l'art, des conditions de son édification, des appareils qui en permettent la compréhension. Nous évaluons nos repères personnels, nos différences et différents, nous le faisons en grande amitié. Ce point est important puisqu'il a permis d'ouvrir des questions sur et dans mon propre travail d'artiste. Il a levé cette inquiétude qui voulait que toucher à l'intime, dans sa part trop biographique, ne portait que paradoxe théorique. Ce texte permet d'ouvrir à la compréhension du faire-œuvre l'ensemble des événements, des constructions, des rencontres qui y participent.

Des souvenirs, même lorsqu'ils s'étoffent, ne constituent pas toujours une autobiographie. Et ceci n'est est certainement pas une, pas même pour les années berlinoises dont il est uniquement question ici. Car l'autobiographie a trait au temps, au déroulement et à ce qui fait le continuel écoulement de la vie. Or il est question ici d'espace, de moments, de discontinuité. Car même si des mois et des années surgissent ici, c'est sous la forme qu'ils ont à l'instant de la remémoration. Cette étrange forme - qu'on l'appelle éphémère ou éternelle - n'est en aucun cas la matière dont elle est faite, celle de la vie. ${ }^{1}$

3 Étrange forme que celle de la mémoire, de la mémorisation d'une absence dont on ne connaît ni la longueur ni l'épaisseur. Étrange espace que celui qui cherche à identifier un moment que l'ici et maintenant pense fondateur.

4 Le texte de Jean-Louis Déotte pose, dans mon travail de peinture (et je pense de poésie), une interpellation à propos du coma, ou plutôt d'un acte fondateur qui ne serait pas (que) de mémoire. Cette hypothèse stimulante inverse en quelque sorte le prédicat de l'œuvre comme trouvant ses sources dans la seule histoire, dans l'addition des expériences, dans l'éternel retour.

5 Je vais donc autant revenir sur certains points du texte de Jean-Louis Déotte qu'éclairer, pour moi aussi, les parcours difficiles qui fondent ma pratique de peintre, de poète ${ }^{2}$. Dans ce sens je vais réagir à certaines phrases du texte mais aussi rebondir 
dans ce qu'il faut appeler un dialogue fertile. L'objet reste bien entendu de réfléchir sur les fondements de la création, sur ce moment et ce départ mystérieux qui fondent toute chute dans le choix de la création.

\section{Traces, œuvres sans traces}

6 Il s'agit encore de comprendre mieux certains aspects du texte et de revenir sur la terminologie qui, alors, engage des points théoriques nouveaux. J'aimerais saisir ce que signifie ce «peut-on œuvrer sans tracer? ? qui ouvre le texte de Jean-Louis Déotte. Ce que cela évoque dans mon histoire c'est que j'ai choisi de faire trace (par la peinture, le dessin, la gravure, la sculpture, le poème et sûrement le texte théorique) au début de mon aventure, en admettant que je suis alors, à ce moment-là, dans l'enfance ${ }^{3}$ de mon travail. Mais aussi qu'œuvrer c'est s'inscrire dans les traces de la vie qui donne à l'art son sens comme vie pour paraphraser en déplaçant quelque peu le propos de Georges Filliou. Mais il faut préciser de quel moment de mon travail de peintre je parle. Nous reviendrons sur cet accident ${ }^{4}$ qui détermine, pour ma vie et mon engagement d'artiste, un chemin qui, peut-être sûrement sans cela, n'aurait pas existé. Mais dans cette première réaction au "œuvrer sans tracer" je suis déjà dans un moment où ma peinture était fortement élaborée, je veux dire dans un moment où j'avais déjà engagé de faire de la peinture, d'y réfléchir et d'une certaine manière d'y refléter ma vie, ou la compréhension que j'en avais à ce moment-là. Cette première période où j'ai conscience de ce que peut être la peinture, je l'appelle " période blanche » où, selon un protocole sur lequel je suis maintes fois revenu, je tentais une conciliation entre écrire (mon travail poétique) et peindre (produire des surfaces). Cette conciliation (qui n'était pas une réconciliation) voulait seulement répondre à un besoin de cohérence, ce qui somme toute, dans ces époques-là, résonnait comme un topos de la contemporanéité. J'ai dit alors que mon geste (s'écrivant de gauche à droite, tout comme j'écris) prenait la forme d'une écriture sur la surface ${ }^{5}$. J'ai abandonné plus tard un tel projet parce que ce que me montrait mon travail de peinture c'est une solidarisation entre le fond et la forme ${ }^{6}$. C'est-à-dire en quelque sorte l'impossibilité de déterminer l'avant et l'après, le dessous du dessus. Si cette énonciation pose encore des points théoriques dans les termes de l'époque (conceptuel, marxiste, celui du tout montrer, etc.) il n'en reste pas moins vrai que j'y vois une posture nouvelle et singulière qui me fait dire aujourd'hui que je ne fais ni de la peinture abstraite ni de la peinture figurative, et que j'opterais plutôt pour le terme de concret (konkret) tout comme aujourd'hui en parle Pierre Soulages. Cette précision, et pour bien saisir la proposition de Jean-Louis Déotte, fait que dans l'apprentissage de mon art (enfance) j'en suis encore à l'écriture d'une trace mais que lentement je suis conduit à des opérations (celles que fait la peinture d'aujourd'hui sans que je ne maîtrise rien, ou peu, ou moins) où la décision de garder ce qui advient est le plus fondamental. 


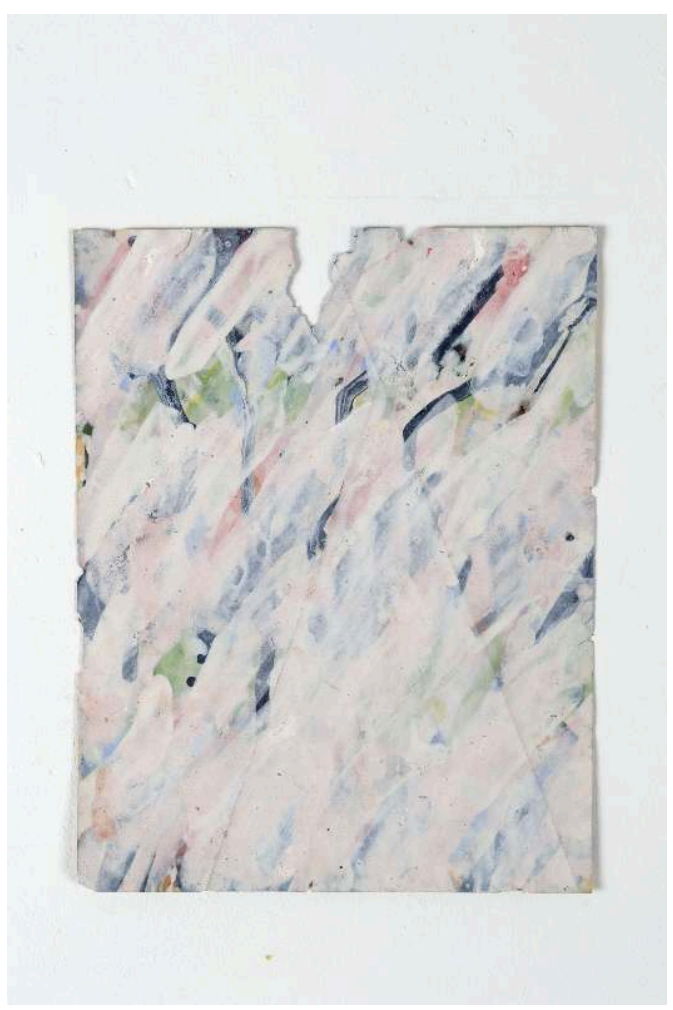

P-79-14, $27 \times 21 \mathrm{~cm}$, vinyl sur papier, 1979

Photo Hess

\section{Se faire signifier le réel dont nous ferons autre chose}

7 Je pense à des remarques du peintre allemand Helmut Dorner.

Beuys hat gesagt "Denken ist Plastik». Ich könnte auch sagen, "Denken ist Malerei » oder «Denken ist Form ». Wenn tatsächlich eine neue Form entstanden ist, ist es das Wissen um diesen kleinen Schaltmoment, an denen Form sich generiert. Form heißt auch Existenz von etwas Neuem. Und das Wissen um die Labilität und die Feinheit und die Zufälligkeit, das ist das Spannende und das große darin. Das kann man natürlich genauso auf sich selbst beziehen, auf den Körper oder den Geist, wo ja auch nur kleine Verschaltungen die Gedanken schaffen. Früher hatte ich gedacht, die Malerei ist eine Art endloses Denken, das nur durch Schlaf unterbrochen wird, und zum Teil ist das ja auch so. Aber häufig denkt man ja so vor sich hin: Ich schreibe etwas, gehe aufs Klo, denke an meine Frau, denke ans Geld, denke an der Schule, und eins ist mit dem anderen verknüpft und andererseits ist jedes isoliert. Aber dann entsteht der Gedanken, der mehr ist, der eine höhere Organisationsform, eine höhere Erkenntnis beschreibt. So ähnlich sehe ich das auch in der Malerei. Es lässt sich einerseits unterverschlüsseln: Das ist die Farbe, das Material, die Malerei, aber dann ist da das Bild, und das ist dann die Einheit. Das Bild ist dann tatsächlich jenseits aller Reflexion eine neue Existenz. ${ }^{7}$

Beuys a dit « Penser est sculptural ». Je pourrais aussi dire, " penser est peinture », ou "penser est forme». Lorsque effectivement une nouvelle forme est advenue, c'est le savoir de ce petit moment déclencheur, au cours duquel la forme se génère. Forme signifie aussi existence de quelque chose de nouveau. Et la connaissance de l'instabilité et de la finesse et de l'accidentel, c'est ici que se trouve le plus 
passionnant et le plus important. On peut bien entendu l'appliquer à soi-même, pour le corps ou l'esprit, où les pensées ne sont produites que par de petites connexions. Avant, je pensais que la peinture était une espèce de pensée sans fin, qui n'était interrompue que par le sommeil, et cela, en partie, est vrai aussi. Mais souvent on pense ainsi pour soi, dans une sorte de distraction: j'écris quelque chose, je vais aux toilettes, je pense à ma femme, je pense à l'argent, je pense à l'école, et chaque chose est re-liée aux autres; et en même temps chaque chose est isolée. Mais alors apparaît une pensée, qui est davantage, qui décrit une forme organisée plus élevée, qui décrit une plus grande connaissance. C'est comme cela que je considère la peinture. D'un côté cela se laisse (dé)chiffrer : cela est la couleur, ceci est le matériau, là c'est de la peinture, mais après, après tout cela, il y a aussi le tableau et c'est alors qu'il y a unité. L'image est alors effectivement endeçà de toute réflexion une nouvelle existence. ${ }^{8}$

Ce qui m'intéresse évidemment c'est l'idée de cette pensée sans fin interrompue par le sommeil. Il s'agirait aussi de penser le coma comme une interruption. Une disruption. Ce serait différent de l'enclenchement, du déclenchement. Une suite d'événements, de constructions culturelles tout simplement interrompues, un temps, et ensuite la poursuite d'un état qui a déjà été! Mais, comme Dorner, je pense que la distraction et les événements qui nous distraient fondent une nouvelle existence. Je peux appeler cela comme une re-connaissance d'une image et/ou d'un tableau non encore vus. Reconnaissance au sens où cela s'agrège de ce que nous possédons avec ce que nous ignorons. Je vois celui qui voit et que je ne reconnais pas totalement. Je dirai plus loin comment cela se traduit dans la décision de faire des peintures dans un sens proche, où l'événement est produit par moi-même puis continué par une non volonté, déclenchement et contre déclenchement, poursuite et interruption. À chaque fois ce qui arrive n'est pas ce qui était prévu, décidé. Il faut ainsi refaire le choix de reprendre ou d'interrompre. Du déchiffrement, puis de l'inconnu. De ces états, de ces situations, tirer le parti d'une re-existence différente (de l'objet et du peintre).

\section{L'ordre des événements}

10 Je ne veux pas aller trop vite dans ma réponse. Je vais donc essayer de donner un ordre aux événements qui m'ont conduit vers la peinture, vers la poésie et l'art en général. Je peux rappeler qu'à l'âge de quatorze ans j'avais bien mal engagé ma vie dans la sphère scolaire. Redoublant successivement la classe de $6^{\mathrm{e}}$ puis celle de $5^{\mathrm{e}}$, je me retrouve à rejoindre un univers, au fond familier, qui est celui de l'abattoir de Colmar ${ }^{9}$, et plus précisément d'un environnement fait de dureté (les ouvriers dans ces lieux sont des gaillards solides, monstrueusement forts, ce qui ne laissait pas de m'impressionner, et toujours dans une sorte de compétition sur les poids à soulever ${ }^{10}$ : des carcasses de porc, de veau, des quartiers de génisse, des sacs de sel, etc. Ce fut un apprentissage où l'expérience du corps, d'éprouver son corps et dans le même temps de pénétrer dans le monde des adultes, se présentait en forme d'initiation, de passage. Un monde bien entendu très particulier qui oscillait entre entraide et jugement d'exclusion. Le deuxième point, qui est évidemment fondamental pour ce qui sera la suite de mon existence, c'est ce rapport à la chair (et ce passage de l'animal vivant à l'animal mort), ce rapport au sang, à la tripe, à la merde. C'est un lieu de couleurs, d'odeurs et de cris. C'est très certainement pour des raisons aussi lointaines que mon regard est dans une plus grande acuité lorsque je rencontre certaines œuvres: le bœuf écorché de Rembrandt, celui de Soutine, les tableaux de Chardin et par-dessus tout le Christ mort 
de Holbein du musée de Bâle. Je suis allé bien souvent dans cette salle du Kunstmuseum de Bâle et suis resté assis longuement devant ce tableau, comme pétrifié, sentant dans le regard et la tête ${ }^{11}$ comment la chair se décomposait.

Revenons à cette expérience de l'abattoir de Colmar. À ce moment-là bien sûr je ne savais rien de la peinture, et si peu de la poésie. Mais à revenir sur cette lointaine période je peux malgré tout saisir des moments de réelles émotions esthétiques. Je ne voyais pas tant la bête qu'on tuait que les traces et les couleurs des chairs, des ventres, les veines, les poils et les soies, les entrailles comme un magma de peinture. Et pour l'école qui avait précédé et pour laquelle j'ai dit que je n'avais pas d'intérêt, il me faut dire malgré tout que j'aimais y faire ces frises colorées et géométriques qu'on nous demandait de faire pour inaugurer un travail d'écriture ou pour le finir. J'aimais aussi les rimes des poèmes appris et plus exactement le rythme qui y courait.

L'accident arrive près de mes quinze ans. Coma. Réveil. Éveil. Je resterai couché près de deux ans. Réapprentissage de la marche qui m'occupera des années durant.

J'ai commencé à peindre et à écrire (de la poésie) dès mes seize ans. À ce moment-là je ne savais pas ce que signifiait peindre. Je m'inscrivais dans une histoire que je ne connaissais pas, dans un auto-apprentissage qui sera aidé plus tard par quelques artistes rencontrés puis par des études universitaires et un passage aux Arts décoratifs ${ }^{12}$ de Strasbourg. Cette période présente peu d'intérêt si ce n'est que dessiner ou peindre se fondait sur du réel observé, ou des revues pour les adolescents. Donc sur des modèles et sur la croyance que s'appuyer sur le réel était le lieu de l'art.

Pourtant, ce qui m'a décidé à faire de la peinture, c'est une expérience au cours de mon hospitalisation. Juste après la sortie du coma. Cette expérience je ne l'ai comprise que très tardivement. La nuit, dans la solitude et la douleur, j'avais constaté que lorsqu'une ambulance entrait dans l'enceinte de l'hôpital, l'alternance de la lumière bleue du gyrophare projetait au travers des stores lamellés, un gris qui se colorait d'orange ${ }^{13}$.

Je ne savais pas à ce moment-là ce que signifiait cette " apparition ». Je sais aujourd'hui bien entendu que l'effet cinétique, les contrastes (celui-ci simultané) ne suffisent pas à faire peinture. Mais force est de constater que je viens à la peinture, que je renais à la vie par un trait de lumière, par un effet optique.

\section{Découverte de la peinture. Cela commence à l'hôpital}

Tracer maladroitement des lignes. Copier maladroitement des têtes de vedettes de la chanson (je me souviens de Sylvie Vartan, des Beatles). Croire qu'une certaine ressemblance participait de l'art. Ma mère m'offre une boîte de couleurs à l'huile (que je possède toujours). C'est l'irruption de la couleur, et la croyance toujours ingénue que l'huile fait la peinture et le peintre. En disant cela je souris en pensant à Duchamp.

Cette période, que j'appelle ma période couchée $^{14}$, fut considérable en termes d'expériences de la douleur, du corps, de l'humiliation, de l'apprentissage de l'émancipation, de ma révolte d'une manière générale. Jean-Louis Déotte, dans nos entretiens, me demande de préciser la teneur de cette expérience parce qu'il y voit une dimension importante qui est celle de l'appropriation. Je vais donc m'arrêter sur quelques points dont je sais aussi qu'ils sont fondateurs dans ma démarche et mon parcours. 

reconnaître, se comprendre dans mon récit.

\section{Sortir du réveil}

Se réveiller sans comprendre la douleur qui saisit. Intense, corps entier vrillé, douleur dans le corps entier. La tête, la pensée submergée par la douleur. Demande que ça s'arrête. Demande de mourir.

Lorsqu'on m'a sorti du service de réanimation au bout de plusieurs semaines, les infirmiers m'ont conduit dans un autre service. Salle commune (une trentaine de lits, beaucoup de bruits, des visiteurs autour de presque tous les malades ou traumatisés présents) : venir d'une solitude infinie (d'un lieu clos) pour une sorte de hall de gare fut une épreuve terrible, et encore aujourd'hui je la pense inhumaine. Le deuxième acte de ce changement de service fut qu'on me changea de lit. Les lits appartiennent au service et doivent donc y revenir! De ce transbahutement je crois que j'ai mis trois jours à me remettre. Douleurs accrues, attelles déplacées en même temps que mon corps. Je crois que j'ai crié et gémi longtemps. Aujourd'hui encore de la colère me vient de ce moment.

Quelques jours plus tard, sur l'insistance de mes parents, je me retrouvai dans une chambre à deux lits, je revins dans un calme qui m'était nécessaire pour me reconstruire. Il me semble que mes parents n'avaient guère le moyen de financer une telle entreprise. Je ne sais toujours pas comment ils s'y sont pris. L'amour fait bien les choses.

Quelques informations sur l'état de mon corps. Fracturé de haut en bas: grande hypophyse du calcanéum ${ }^{15}$, tibia, péroné, fémur, côtes, bras, maxillaire, face enfoncée. L'état d'un corps qui ne bouge pas. Comme paralysé.

$\mathrm{Au}$ réveil, au retour, ne pas pouvoir parler, ne pas pouvoir toucher son corps, ne pas comprendre ce qui s'est passé. Juste une peur qui ira croissante d'entendre ma respiration au niveau de ma gorge. Une canule. Je ne savais pas, je ne comprenais pas.

Peur encore lorsque la personne dans le lit à côté, que je ne voyais pas, hurlait, commençait à renverser des objets (j'entendais le bruit) et disait vouloir me tuer. Crise de délirium tremens. Les infirmiers sont intervenus, l'ont attaché. J'étais attaché aussi du bras gauche afin de ne pas arracher les drains, les tuyaux qui me transfusaient. Peur puisque je n'aurais pu me défendre. Ce moment contracte l'envie de mourir pour que s'arrête la douleur, et la peur de mourir parce que j'étais à la merci d'un autre.

Se succéderont de multiples opérations, ostéotomies.

\section{Anecdote fondamentale}

J'avais la mâchoire cassée et décrochée. Je suis resté ainsi pendant six mois. Les chirurgiens avaient considéré que l'urgence concernait les jambes et qu'il ne fallait pas ajouter de chocs au cœur qui manifestement avait déjà pas mal pâti. Ce n'est donc qu'au bout de six mois qu'on réduira cette fracture de la mâchoire. Si je parle de cet événement, c'est que je ne savais pas (à cette époque les chirurgiens ne parlaient pas aux malades!) ce qui allait m'arriver. Anesthésie locale. J'entendais parfaitement le dialogue des deux chirurgiens et l'évocation des deux techniques possibles pour une 
telle réduction. Celui qui ne connaissait pas la technique par l'extérieur demanda à l'autre de lui montrer. Sentiment du cobaye. À l'issue de cette opération, je me retrouvai avec la bouche totalement close. Ils m'avaient attaché les dents, m'en avait retiré une au passage pour pouvoir me nourrir de liquides divers (jus de carotte, jus d'orange, jus de tomate et autres soupes concoctées par l'hôpital, autrement dit un programme gastronomique peu ragoûtant) le temps de la consolidation. Parler dans ces conditions fut difficile ${ }^{16}$, et l'incorporation de tout aliment autre que liquide donné par une pipette par le passage de la dent manquante fut une autre expérience où l'appropriation de son corps par la parole, par l'ingurgitation fut donc quasiment impossible. Dans mon souvenir cela dura trois mois.

N'étant aucunement autonome, je ne pouvais ni me laver ni m'occuper de ce qui relève de l'intimité la plus grande. Au départ j'ai vécu cela comme une totale humiliation, puis petit à petit cela m'est devenu égal, et bon nombre de mes pudeurs sont tombées.

Muet un temps, impotent un temps, je n'avais qu'une hâte, c'était de pouvoir être debout, ne plus m'arrêter, de croquer (dans) le monde.

La première fois où l'on m'a assis au bord du lit et où l'on a descendu mes jambes vers le sol, j'ai failli perdre connaissance comme si tout le sang qui était en moi affluait brusquement dans les jambes. Plus tard, la première fois où j'ai posé le pied au sol, la douleur était si grande et je crois que ça n'a pas duré plus de trois secondes. Réapprendre à marcher comme un enfant, forcer, se dépêcher d'avancer. À chaque opération (nombreuses jusqu'à aujourd'hui), un sentiment de lutte et souvent de découragement.

L'art, dans son exercice, m'a toujours permis de dépasser cela, jusqu'à l'oubli de la douleur d'une certaine manière.

\section{Peindre}

31 À dix-huit ans et grâce au permis de conduire, mes parents me mettent à disposition une vieille dauphine qui me permit d'aller au motif. Moments d'intense liberté où, muni d'une toile, d'un chevalet portatif, des couleurs, m'installant dans les hauteurs des vignes, des collines, je ressentais au fond cette liberté dont tant d'années m'avaient privé. Peindre sur le motif.

J'ai donc commencé par des paysages assez matiérés, plutôt colorés.

J'ai ensuite fabriqué des espaces maçonnés à la manière de Nicolas de Staël, de Poliakoff que j'aimais à l'époque.

Dans l'enfance de mon travail, je posais des signes (gestes) de gauche à droite, et parfois de droite à gauche dans des espaces matriciels très fortement influencés par Phil Glass et Terry Riley. Je partais d'une couleur dont je conservais un résidu que je transformais par une autre couleur. À chaque transformation je posais en quelques lieux de ma peinture la couleur élaborée. Je construisais ainsi mon tableau dans une considération de continuité, de construction presque mathématique, mais aussi dans une discontinuité assumée ${ }^{17}$. Donc de l'écriture. J'ai appelé cette série «blanche ». J’ai pensé longtemps que cela avait à voir avec la blancheur des draps, ce linceul de la douleur, mais je constaterai, plus tard, que «blanc » est l'avers du trou noir dans lequel j'étais plongé. 
Je pense que ce coma définit un espace assez proche de celui que décrit Tony Smith et qui le conduisit à réaliser «Die ", ce parallélépipède au format de son corps. En effet, le sentiment de revenir à la vie (consciente) participe d'un autre sentiment, celui d'être accompagné d'un espace dans lequel on a vécu, sans lumière, mais pas sans perception. Bruits ? Odeurs je ne sais pas, mais peurs considérables face à un monde perçu mais non vu. C'est l'expérience de cet espace qui me conduit à parler de Raum, de cette force qu'ont les lieux pour s'insinuer en nous, pour nous faire toucher (mentalement sûrement) ce que le corps ne peut appréhender. Je ne pouvais pas bouger, or la perception était totalement accrue. Le neurobiologiste Le Bihan nous apprend que le fait de perdre des neurones n'est pas bien grave si nous développons ce qu'il appelle le réseau, et, manifestement, dans ce moment du corps souffrant, les conditions de survie extrêmes ont exacerbé mon acuité et mobilisé un voir qui se faisait dans un noir absolu, ou plutôt un noir d'une épaisseur incomparable face à celui que nous croisons habituellement.

\section{Suite Art Press 3}

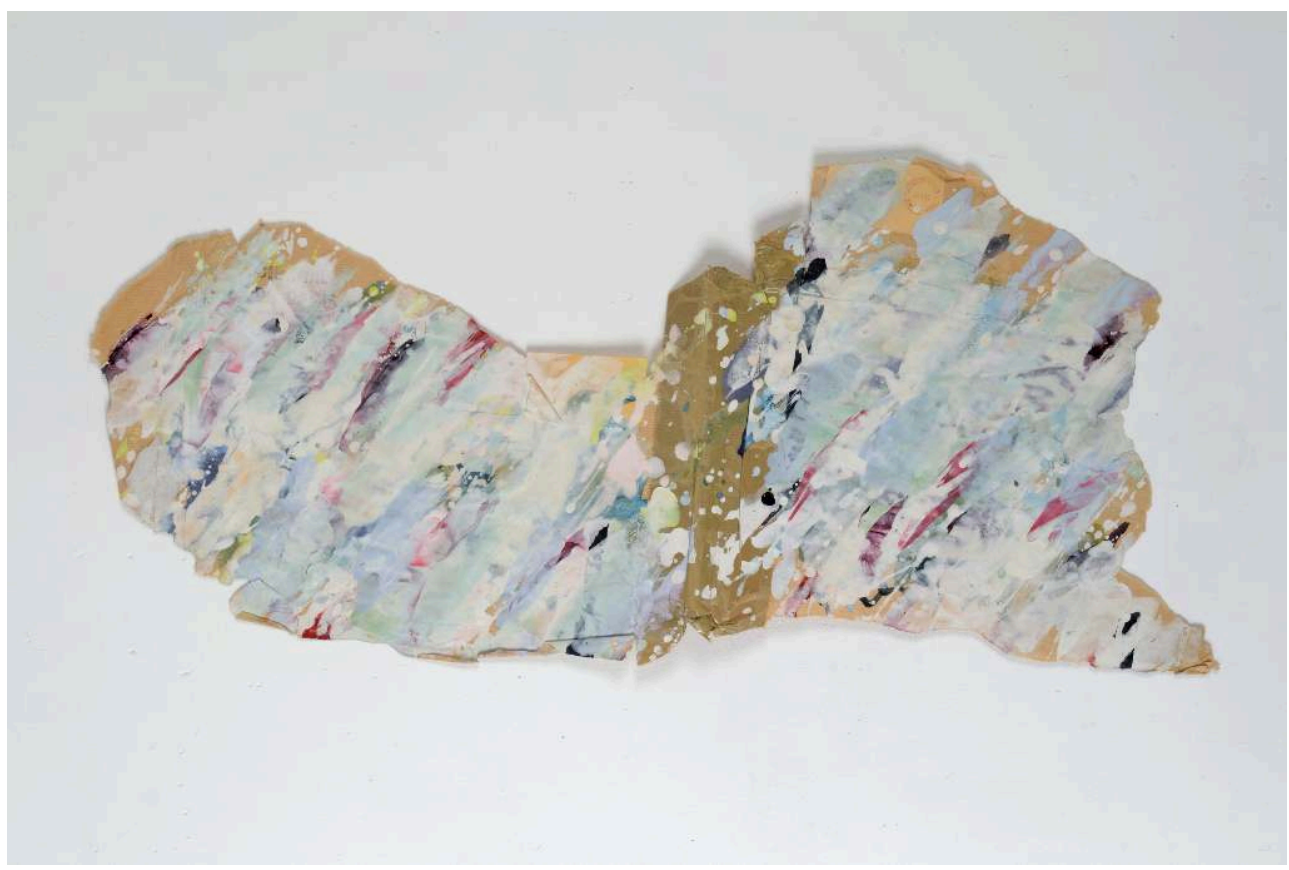

$34,5 \times 76 \mathrm{~cm}$, vinyl encre scotch sur papier kraft, 1983

Photo Hess

\section{Le protocole comme espace, comme chemin, comme cordon}

Aujourd'hui, j'ai conservé le protocole coloristique (décrit ci-dessus) mais en le complexifiant: plusieurs couleurs se transforment en même temps. Je me demande parfois si ce n'est pas une autre forme de la palette des peintres (qui faisait déjà tableau) en considérant un étalement de la couleur en soi. Dans mes pots (ou seaux) elle est toujours séparée et singulière. La procédure de dépose de la couleur a quelque peu changé. Je pose souvent une couleur très liquide (cela a son importance) à côté d'une autre et je laisse faire le mélange, la solidarisation hasardeuse. Certaines couleurs sont 
plus fortes que d'autres, parfois c'est la quantité qui l'emporte, mais pas toujours. Les rouges sont de sacrés guerriers. Je fais ce travail sur plusieurs types de surfaces : du bois, de la toile, du tissu non préparé. Ainsi la couleur peut chercher la couleur contiguë ou imprégner le support, se diffuser, s'étaler, se contracter.

Aujourd'hui toujours, ce chaos presque organique dans sa dispersion est contrecarré par une construction très volontaire, une sorte d'architecture qui ne compose pas avec la couleur, qui ne la compose pas mais qui se donne comme architecture et que la couleur peut contredire, cacher.

Le hasard est la chose que je reconnais, que je conserve. Au fond, je m'intéresse à un lâcher-prise, à une observation des maniements de la couleur tout comme un rêveur extatique pourrait le faire de la nature. Ce qui se joue ici, dans le lieu de l'atelier, c'est une forme de synthèse de ce qui se joue dans le monde, mais qui échappe, nous échappe dans le monde, et qui dans l'atelier se dévoile strate après strate, diffusion après diffusion, et ainsi de suite.

Je crois que même quand la peinture est achevée ou considérée comme telle par moimême, elle reste dans un état de vie, de continuité qui lui donne son mystère, sa force, sa vitalité.

40 Ce sont en quelque sorte un réveil continu de la couleur, des potentialités du tableau et des observations du regardeur qui se déroulent. C'est l'après coma.

41 Me viennent les mots de Beckett au tout début de l'ouvrage L'innommable :

où maintenant? Quand maintenant? Qui maintenant? Sans me le demander. Dire je. Sans le penser. Appeler ça des questions, des hypothèses. Aller de l'avant, appeler ça aller, appeler ça de l'avant. Se peut-il qu'un jour, premier pas va, j'y sois simplement resté, où, au lieu de sortir, selon une vieille habitude, passer jour et nuit aussi loin que possible de chez moi, ce n'était pas loin. Cela a pu commencer ainsi. Je ne me poserai plus de question. ${ }^{18}$

Et

Qu'est-ce qui me fait pleurer ainsi ? De temps en temps. Il n'y a rien ici qui puisse attrister. C'est peut-être de la cervelle liquéfiée. Le bonheur passé en tout cas m'est complètement sorti de la mémoire, si tant est qu'il y fût jamais présent. Si j'accomplis d'autres fonctions naturelles, c'est à mon insu. Rien ne me dérange jamais. Néanmoins je suis inquiet. Rien ne change ici depuis que je suis ici, mais je n'ose en conclure que rien ne changera jamais. Voyons un peu où ces considérations conduisent. Je suis, depuis que je suis, ici, mes apparitions ailleurs ayant été assurées par des tiers. Pendant ce temps tout s'est passé dans le plus grand calme, l'ordre le plus parfait, hormis quelques manifestations dont le sens m'échappe. [...] D'où me viennent ces notions d'ancêtres, de maisons où l'on allume, la nuit venue et tant d'autres ? ${ }^{19}$

43 J'ai chié, pissé sûrement, j'ai mangée ${ }^{20}$ mais comment? Je n'en ai aucun souvenir. Peindre, c'est fabriquer du souvenir, lointain, à venir, pour plus tard, pour construire une histoire à soi.

C'est donc bien d'une appropriation qu'il faut parler, celle du corps, d'une expérience qui puisse se transformer en forme, en sens.

$\mathrm{Au}$ début de son texte, Jean-Louis Déotte questionne: "N’y a-t-il pas des milieux culturels trop riches?». Ce milieu il a fallu l'apprivoiser, l'approcher, le dire dans un dit incertain, dans une appropriation presque non culturelle. Il a été construit dans un dialogue et un débat permanent. Le groupe du Faisant et avant lui le groupe Attitude furent des ferments de nos évolutions individuelles, de nos positionnements politiques, 
communistes à l'origine et socialistes pour d'autres, d'ailleurs sources de disputes et de frictions. Mais aussi, et je crois cela fondamental, le groupe remplaçait à cette époque la carence en lieux d'exposition pour l'art contemporain, la carence d'un vrai débat (et de la hauteur d'un débat sur la signification de l'art, ses limites, son champ d'opération et bien entendu ses réceptions). Et davantage encore cela remplaçait les enseignements désuets, souvent académiques, pour et par une forme d'apprentissage ensemble. Nous pouvions ainsi observer, au plus près de la création, comment se fabriquaient les forces et les faiblesses, et nous pouvions, davantage encore, considérer que les faiblesses devenaient des forces. Ainsi, le métier de peindre était totalement bouleversé pour un savoir-faire nouveau qui n'était pas moindre que celui qui se transmettait de manière inébranlable. Il faut considérer alors une ouverture à l'expérience, une exploration des limites, parfois sans limites.

Dans la perspective que prend Jean-Louis Déotte, je dois interroger ce que l'expérience de l'accident et encore davantage du coma peut avoir de fondateur dans l'immersion de l'apprentissage ensemble, d'une espèce de percée ${ }^{21}$ ensemble pour apprendre au commencement.

Indéniablement je n'étais pas encombré de savoirs qu'il fallait connaître (et que d'autres connaissaient et connaissent). C'est, dans le même temps, une grande ingénuité et une difficulté pour le dialogue avec les autres. C'est dans ce sens sûrement que je travaillerai plus tard comme un forcené pour apprendre la culture, toute la culture. Le champ vierge en quelque sorte oblige à construire davantage, et, pour ce qui me concerne, à comparer la culture (extérieure) à l'expérience propre (intérieure).

Un point, mais que certainement bien des peintres pourraient convoquer, concerne l'amnésie qui se fait à un certain moment du travail de création. J’ai déjà développé ce point en parlant de la cécité (texte réalisé à la demande d'Anne Pétrequin et publié dans la revue L'Ollave Préoccupations $\mathrm{n}^{\circ} 30$ ). Le travail dans l'atelier se fait à partir du travail déjà-là, des choses en cours, voire des peintures que je crois devoir démarrer. Ce travail se fait dans une certaine distraction et souvent j'interpose des faires qui n'ont rien à voir avec la peinture. Ranger, déplacer un objet, balayer l'atelier, etc., comme s'il s'agissait de différer le moment important. De la même manière, même pour la peinture en cours (il y en a toujours une qui prend plus d'importance que les autres, les autres servant à ce moment-là justement à la mise au point, à la contribution de celle qui mène la danse), je fais des gestes presque irréfléchis, je pose de la couleur, je fais couler, je fais un pli rapidement, et puis vient un moment où cela s'accélère, où tout s'accélère: décision de l'urgence, choix des couleurs, dépose de celles-ci, jusqu'à un blanc où ni le corps ni l'esprit ne dirige la peinture dans ce faire. Je pourrais dire de ce moment, d'où l'on ne sort que sonné, que c'est un autre corps qui se fait jour, que saisit la peinture, qui la dirige. Ce n'est donc pas un coma, bien sûr, mais c'est un espacetemps sur lequel on ne peut rien dire, on ne fait que constater ce qui s'est passé, parce que "quelque chose " s'est passé entre le faire-œuvre, l'œuvre et le corps. Ce qui s'absente dans ce moment-là, c'est la volonté de maîtrise. Le retour au réel est évidemment douloureux. Le réveil du coma est lui aussi douloureux parce qu'il dit que quelque chose s'est passé et nous ne savons pas quoi. 


\section{Parler de l'encombrement et du fait que je trébuche, que je tombe} d'un peintre, Jean Dubuffet, comme le montre bien Céline Delavaux ${ }^{22}$ ) ont une mémoire (même s'ils ont perdu des fragments de leur réalité vécue). En fait, il faudrait dire qu'ils ne sont pas inscrits (ou ne se sont pas inscrits dans le champ de l'art savant ou officiel) pour de multiples raisons: manque d'informations, manque d'intérêt, incompréhensions, n'imaginant même pas que cela existe, faisant face à de nombreux problèmes psychologiques voire à des dérèglements multiples, autocentrés sur leur propres créations ou productions artistiques. À noter que l'autocentrement n'est pas l'apanage des artistes de l'art dit brut!

Je ne tiens pas à développer ici ce qui m'intéresse dans ces arts en marge, à la marge, dans ces questions qui relèvent aussi de la maladie, de l'aliénation, de l'angoisse, de la périphérie, de la révolte, mais je veux juste préciser que le répertoire des formes est ici souvent considérable, surprenant, et surtout que le lâcher-prise, cette donnée qui ne répond pas aux diktats d'un certain art contemporain, peut être salvateur. Dans quel sens salvateur, nous sauver de quoi en effet? Du formalisme, du diktat de la cohérence, de la continuité, de la réponse que devrait l'art à la seule partition contemporaine. Mais, en disant cela, je ne refuse pas la forme et les concepts qu'ont élaborés un certain nombre d'artistes. Je pense à Opalka ou à Bruce Nauman qui savent si bien allier cohérence et hétérogénéité. Il faut dire aussi que l'hybridité des œuvres d'art brut marque aussi pour moi un intérêt pour l'excès, pour une forme de violence faite à ce qui est établi, pour des questionnements qui interrogent les ténèbres, les faces cachées, les incertitudes, les fantômes.

51 La lune ne nous montre qu'une face depuis la nuit des temps. Il en est de même des œuvres. Il faut que nous nous mobilisions autour d'elles, que nous inventions des voir, des procédures, des machines pour les saisir en totalité en sachant bien qu'en fin de parcours toujours quelque chose échappera. C'est ce qui échappe alors qui ouvre encore et encore nos interrogations, qui modifie nos perceptions, qui oblige à recommencer. Chaque œuvre, tout comme la face cachée de la lune, nous dit que cela existe, nous invite à aller voir et nous donne cette liberté de l'interprétation.

\section{Parler de l'absence - de s'absenter du réel}

Ce que décrit Elsa Morante et ce que commente Jean-Louis Déotte peut et doit se rapprocher de ce moment où, dans l'élaboration d'une œuvre, l'artiste perd tout contact avec le réel. Bien sûr pour un contact avec un autre réel. C'est un moment de perte où l'œuvre se trouve. "Se trouve » signifie autant «se rencontre " que l'œuvre qui s'élabore dans un langage autre que celui que nous utilisons quotidiennement. Cet autre n'est pas une chose obscure, indicible, mais bien une forme qui approche l'autre pour le remettre au monde, pour le faire revenir au réel qu'il pourra alors appréhender. D'une manière triviale je dirais que c'est même la seule possibilité de com-prendre le réel.

Sans vouloir revenir sur la différence que fait Lacan entre réalité et réel il s'agit de marquer ${ }^{23}$ (comme on délimite un territoire) le lieu où l'on peut penser le réel. Ce lieu 
est la réalité concrète qui nous heurte mais aussi celle qui s'élabore dans l'œuvre. Ce lieu finit par dire sa porosité, ses extensions possibles. Le lieu est comme un coma où ni les yeux ni les mains ni l'oreille ne peuvent affirmer une vérité, mais où la combinaison des organes fait comme un orifice que j'appelle Raum. C'est d'un toucher qu'il s'agit, d'un toucher aux confins du mutique. Sûrement que les artistes de l'art brut sentent cela. Dans le champ analytique, rhétorique qui est aussi le nôtre nous mettons des dires, des socles, des définitions sur le ressentir. Sûrement que des artistes singuliers qui sentent ne savent exprimer que ce sentir. Peut-être que cela les rend plus heureux, plus apaisés et aussi parfois davantage dans la douleur.

54 La répétition, le remplissage de l'espace (on parle d'une peur du vide) peuvent être catégorisés psychologiquement mais alors il faudrait comparer avec d'autres œuvres, celle d'Opalka qui fonde une partie de sa démarche sur cette pendule qui s'arrêta pendant son enfance, il faudrait confondre la réalité et la mythologie de Joseph Beuys, il faudrait revenir sur l'affichage compulsif peut-être de Hane Darboven, ou sur le développement sans limite des boules chez Jean-Luc Parent, et aussi sur les logogrammes s'enchaînant les uns les autres chez Christian Dotremont qui fit cette découverte, avec Cobra, de l'antispécialisation.

Voyons ce que dit Beuys de son expérience :

Puis quand j'ai été abattu et qu'il a été impossible de me retrouver à cause des fortes chutes de neige, s'ils ne m'avaient pas découvert dans la steppe, par hasard en gardant leurs moutons ou en poussant leurs troupeaux de chevaux [...] Puis ils m'ont emmené dans leur hutte. Et là toutes les images que j'ai perçues je les ai perçues sans en être tout à fait conscient. Je suis pratiquement revenu à la conscience au bout de douze jours seulement et je me suis retrouvé alors dans un hôpital militaire allemand. Mais là-bas toutes ces images m'ont entièrement, disons imprégné. Sous forme figurée peut-on dire. Les tentes, ils avaient donc des tentes de feutre, tout le comportement des gens, l'histoire de la graisse, c'est comme... une odeur généralisée dans les maisons et le fromage et la graisse, et le lait, le lait caillé - tout ce qu'ils manipulaient, tout cela je l'ai complètement intégré en moi, j'ai vraiment vécu cette expérience. ${ }^{24}$

L'inconscience, le coma laissent ainsi filtrer, en accentuant l'impact de la mémoire. Au fond, ces advenues d'odeurs, de bruits, de chocs et peut-être même de murmures se donnent symboliquement dans une confusion avec (et dans) le corps. Je n'ai pas travaillé avec l'éther mais cette odeur me vient toujours aujourd'hui lorsque je me rends dans un hôpital (où ce produit n'est plus utilisé). Les pas feutrés, ce feushhh des paroles partagées continuent de me submerger, et nul doute que mon travail, sur la langue et sur les mots, retraduit ${ }^{25}$ cette membrane entre le réel et l'inconscience, comme un bégaiement qui cherche à produire du sens par ce seul moyen. 
L'espace de la langue

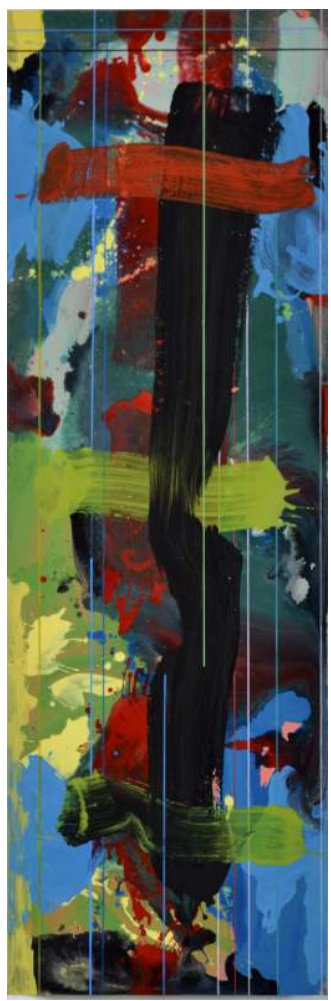

$155,2 \times 50 \mathrm{~cm}, 2011-2013$ Photo Hess

\section{Aveugle. Sentir dedans et dehors simultanément}

57 Ce qui revient souvent dans mon texte, mes réponses, mon expérience mais aussi dans celles de nombreux peintres, c'est l'aveuglement, ne pas voir, finalement autre terme de l'éblouissement. Cette absence de couleur, de lumière reste pour le peintre que je suis une expérience fondamentale, et sûrement assez souvent revécue. Le coma est un ventre de la mémoire, une sorte de point invisible. Je sais aujourd'hui qu'un point que l'on trace sur un papier ou sur une toile sait son espace, c'est-à-dire sait le développer, sait s'y promener.

J'ai, et je l'ai rappelé plus haut, écrit un texte sur l'aveuglement à la demande d'Anne Petrequin, artiste et théoricienne, au moment de la rédaction de sa thèse.

59 Je donne ce texte comme un autre argument de ce que déclenche positivement l'aveuglement, la perte de la conscience. Et je me dis qu'au moment où le photographe déclenche, il ne voit pas, ou plus, mais il va voir mieux.

\section{Expérience de cécité passagère ${ }^{26}$}

Que serait l'instant d'aveuglement où ne pas voir (quoi?) pourrait cependant se décrire. L'aveuglement est comme un oubli. La vraie question est de mesurer si cela est volontaire, provoqué, ou survenu à nos dépens. 
61 D'une autre manière cela questionne l'art que l'on fait, que l'on envisage, dont on a l'ambition. Cela pour signifier que s'il s'agit de correspondre au monde (environnant) nul besoin d'aveuglement. Au contraire yeux bien ouverts et mimésis parfaite. Mais bien sûr les choses ne sont pas aussi simples.

62 Si l'ambition est de construire un monde face au monde alors évidemment les choses sont bien différentes. Que sont d'ailleurs les choses? L'objet du travail, ses fondements, ses outils, sa visée?

63 Avant que de poursuivre j'aimerais revenir sur la question elle-même et sur les mots qui la thématisent: aveuglement, cécité, éblouissement. Il me semble qu'ils ne fonctionnement pas sur le même mode. L'aveuglement et l'éblouissement sont provoqués par un objet extérieur (la lumière, un manque de lucidité face à ce qui arrive). La cécité est un état.

64 D'autre part, pour relater une expérience propre de ce type liée au travail de création, il faut rappeler que nous portons (aussi) en nous une expérience du monde, et des commentaires sur le monde, et donc du voir ou du non-voir le monde.

65 Je rappelle pour mémoire (et de mémoire) que Simon Hantaï dit qu'« il faudrait crever les yeux des peintres » (pour revenir à la causa mentale bien sûr) ; qu'Olivier Debré disait « je peins en aveugle » (il étendait ses toiles en bord de Loire et le soleil faisait miroir de lui-même sur l'huile brillante du peintre : il ne reste alors que l'attention au geste, au corps comme une expérience nue).

66 Il faut se souvenir ensuite que la plongée dans un inconnu de nous-mêmes, un inconnu de l'art fait de l'artiste ce fouilleur aveugle qui déniche le non su, un ailleurs qui sera d'ailleurs peut-être indicible. Cette plongée participe de cette construction du monde où il faut gratter la terre sans lumière, au fond d'une caverne peut-être. La lumière serait au bout du voyage, de l'expérience. Elle éclairera enfin jusqu'à l'éblouissement.

67 La part inconnue est aussi ce que nous projetons, un territoire en quelque sorte à explorer où nous serions autre, neuf en quelque sorte. Je n'ose dire vierge. Cette part qui relève de la naïveté est cependant ce qui donne du sens à la poursuite du chemin.

68 Je suis peintre et je fais ce que font les peintres: je superpose, je sédimente de la couleur, je trace la couleur, je malaxe des pâtes de consistances différentes. Je fabrique une surface qui porte toutes les couches préalables et qui bien sûr est portée par elles. Ce premier point marque l'idée d'une tension, d'une articulation (invisible peut-être) qui donne la force à la couleur (à la surface, à la matière). Je note au passage que la tentation monochrome n'est pas exempte de la sédimentation (les couches de l'histoire comme un lit qui noue). Je reviens sur le terme d'articulation qui est le lieu d'un lien, d'un seuil et d'un passage. Je vais d'un endroit à l'autre et le lieu où cela bascule n'est pas le lieu où je séjourne. C'est peut-être l'objet-fibule ${ }^{27}$ que Daniel Payot développe.

Il y a donc à chaque instant quelque chose qui disparaît et dont je me souviens, dont je dois me souvenir pour saisir ce que signifie ce que je fais. Dans le même temps cela construit ce que je fais au sens où cela donne le sens même de la recherche. La recherche est donc une chose qui signifie que ce qui se fait n'est pas connu, est tâtonnement, est incertitude. Quelque chose s'insinue en aveugle, et il faut le voir jusqu'à l'éblouissement. L'éblouissement nous avertit de ce qui s'est insinué.

70 Je suis peintre et donc je suis porté par la matérialité de l'expérience de la peinture : le châssis, la toile, le pigment, le liant, l'enduit, le geste, l'étirement, l'arrêt, le recul, la projection. Je suis dans un faire dont j'évalue la progression, dans un faire qui lui-même 
me constitue, qui interroge, qui dévie les intentions initiales. Ce point est déjà de l'ordre de l'aveuglement. L'endroit que l'on vise n'est jamais l'endroit où je vais. Il n'existe pas. C'est à un autre endroit que je suis conduit par l'expérience de la peinture. Il faut le repérer et comprendre s'il n'est pas antinomique avec cette exigence de la peinture.

71 Le travail se fait dans un agglomérat d'informations. Notre histoire, l'histoire déjà présente de la peinture en train de se faire, le monde extérieur, les ennuis, les joies. Tout cela est (d')un encombrement extrême. C'est là, c'est présent, trop présent. Cela doit disparaitre pour ne pas tirer le travail vers l'anecdote, vers l'illustration. L'attention à l'ouvrage y aide. L'attention extrême y aide. C'est un dépouillement. L'encombrement se dépose dans un ailleurs que nous ne solliciterons pas pendant un temps donné.

Cela ne se fait pas volontairement. Les années de travail font advenir ce moment très rapidement. Il y a donc un relâchement, un abandon corrélé par une attention accrue, totale, dirais-je, de l'objet en cours. Je précise que cela se déroule de la même manière pour le travail d'écriture.

Une deuxième phase en quelque sorte se fait jour alors. Il y a perte (au sens de se perdre), il y a des moments (longs parfois) où comme une autre vie se déroule entre le corps, l'esprit et la couleur que trace le geste. C'est une union où voir ce qu'on fait n'a aucune importance. Cette union est sans voir (sans le voir), est aveugle. Cette union est saisie après (plus tard) dans un retour au lieu, aux bruits du lieu, à la vie environnante. Cette expérience est faite à chaque moment de peinture, que quelqu'un se trouve dans l'atelier ou non, que la radio diffuse de la musique, ou que dehors des tronçonneuses rugissent. L'instant où je comprends ce qu'on peut appeler un aveuglement est ce retour au monde, comme d'une hypnose, ou d'un coma (mais conscient). Cela dit (nomme) d'emblée la fin de la séance. Je quitte l'atelier, ou alors j'y fais les travaux d'approche, de préparations ordinaires mais nécessaires, le rangement (mais rarement, je préfère laisser en plan, en l'état, comme pour retrouver, la fois d'après, l'infinie sédimentation, l'incertaine légèreté).

La lumière n'est pas utile pendant ce lâcher-prise.

Il me faut aussi parler de la manière dont je fais ma couleur. Dans un seau, et en aveugle. La question n'évoque pas cela. Cependant cela éclaire sur mes procédures d'aveuglement. J'ajoute du pigment, du liant dans un seau qui contient déjà la couleur (précédente) de la dernière séance de travail. Depuis plus de vingt ans, je fais cheminer (je ne dis pas progresser) la couleur de cette manière. Si j'en suis à une couleur blanche et que mon désir (le projet) est le noir, j'en suis réduit à un chemin (long) qui m'y conduit. J'ajoute du pigment noir, et j'ai un gris, je le dépose sur la toile. J'ajoute du pigment noir dans le gris et j'ai un gris noir, et ainsi de suite. Ce que j'ajoute je ne le vois pas vraiment. Je le sais. Je n'ai pas besoin de voir la couleur que je fais pour connaître la couleur. Le dosage, la quantité, le mélange pigment/colorant liant se fait par ce nœud de l'évidence du corps, du geste, de l'esprit que j'ai déjà évoqué plus haut. Le seul appui sur le tube de peinture, ou sur le colorant, ou encore le poids du pigment pur dans la paume de la main suffisent pour savoir de quoi sera fait la couleur : son intensité, sa coloration, son épaisseur, sa qualité.

Elle se fait dans un noir absolu et s'éclaire dans l'esprit. 
77 Ces procédures attestent d'une manière de se projeter dans l'avenir, dans une incertitude. J'ai rappelé plus haut que c'est une plongée aussi (en soi) mais qui extirpe de l'inhabituel pour un devenir. En voilà l'enjeu. En voilà la réalité. Le retour est donc un moment d'égarement. De quoi s'agit-il ? Qu'est-ce que je vois à partir de qu'est-ce que j'ai fait? C'est le moment où ça (me) regarde jusqu'au vertige. Il faut retrouver un équilibre, une raison, une autre attention. L'aveuglement fait perdre des repères. Ce qui est curieux, c'est que la tension est maximale comme le voir est minimal. Cela n'est guère éloigné du kairos nietzschéen.

78 Je me sens très proche de Michel Sanejouand qui dit: «La peinture telle que je l'entends est une prospection, une exploration vers des zones de sensibilités inconnues $»^{28}$. Inconnu que l'on déchiffre dans les gestes et les noirs de la couleur.

79 Un autre point me semble à noter. À la fin de chaque séance de travail, je constate que mes mains sont pleines de couleur(s), et les bras et parfois le visage. La couleur gicle, la main se pose sur la toile ou s'appuie sur le support. Le pinceau tombe dans le seau, je ne le lave pas et donc il tache la main. Tout cela je le découvre en fin. Je lave alors mes mains, mes bras, mon visage, mais il reste toujours des traces de peinture dans les sillons de la peau. J'ai vu encore récemment la tête que font les gens qui me croisent et qui observent ces séquelles colorées sur mon corps ou sur mes chaussures quand il ne s'agit pas de mes habits. Enfant, j'aimais beaucoup la couleur du ramoneur, et j'ai aujourd'hui encore une tendresse pour ce métier qui porte sur son corps la couleur de la sueur et la suie de son travail. Le noir du ramoneur est comme une nuit qui se promène et que je saisis en plein jour et qui m'aveugle. Le noir du ramoneur quand je le regarde s'abstrait aussi de la blancheur du monde. Le noir chemine alors en moi comme couleur, comme lumière.

80 Il y a quelques années, un texte de Ghérasim Luca, scénographié par Kowalski, fut présenté dans les musées de Strasbourg. Les spectateurs que nous étions étaient assis en une ligne continue le long d'un mur. Le spectacle commençait par une lumière violente projetée sur nous. L'aveuglement fut total et je crois bien que dix minutes furent nécessaires pour entrevoir la scène qui s'était ouverte devant nous. Restait dans la tête le texte du poète, juste le texte, et cette présence rare jusqu'à aujourd'hui d'une expérience (forcée certes) d'être au plus près de la voix, du sens, de la scansion.

81 Si j'évoque ce moment, c'est que l'aveuglement que je saisis dans mon travail de peintre est de même nature. Cela permet au moment ultime où je regarde la toile achevée d'en comprendre tous les termes, tous les linéaments et en même temps d'être abandonné de moi comme si un autre était l'auteur de ce j'ai sous les yeux.

82 Je me rends compte que je n'ai pas évoqué la notion de trouble optique. Elle existe dans les formes picturales que j'utilise depuis l'origine. Une incertitude, des blancs sur blanc, qui empêchent de distinguer véritablement l'espace, et cela jusqu'à la difficulté de lire, de mettre au point. J'en suis le premier expérimentateur. Aujourd'hui, les lignes qui s'entrecroisent provoquent un tel trouble aussi. C'est la saisie d'un moment où alternent la couleur qui vient (vers nous comme un scintillement) et la couleur qui s'absorbe dans la couleur (qui nous tire dans la peinture). C'est un moment de déséquilibre, de basculement. Il va de soi que ce n'est pas l'effet optique que je recherche mais bien l'incorporation du corps dans le voir.

83 Plus je suis proche de ce que je tente, plus la fréquence est grande. L'aveuglement, le trouble tiennent donc aussi à la qualité d'attention que l'on porte à ce que l'on fait. 
D'une certaine manière les causes proviennent de la conséquence. Ça n'est ni agréable ni désagréable. C'est ce qui doit être pour la peinture que j'envisage, que je comprends comme un visage qui se peint.

J'ai débordé la question que vous m'avez posée. Cependant dans toute réponse il y a un aveuglement qui s'éparpille pour approcher ce qu'on essaye de saisir. ${ }^{29}$

\section{Léthé}

Dormir, rêver. Penser à ce fleuve, le Léthé, qui coule sans bruit, qui sépare l'enfer du monde des vivants. Je sais qu'il y eut des bruits comme une source qui traverse le fleuve. Le Léthé est ainsi poreux. C'est un état de sommeil non voulu, non désiré dans lequel il y a un état de veille, puis d'éveil.

Dans le moment de peindre il y a toujours cette sensation de saisir un nœud, une forme en quelque sorte qui s'est produite, fabriquée hors de nous et en nous.

Cette forme, nous la transformons, nous la manipulons, nous la déformons.

C'est ainsi, dans ce retour vers ce qu'on pourrait penser une non présence (mais qui pourtant est une archiprésence ${ }^{30}$ ), que se fonde une part du travail artistique. La question est de vérifier si ce que nous apprenons dans le monde éveillé, conscient, n'est pas juste la part de prudence, de se sécuriser face à l'abîme qui toujours renvoient (à) la forme générique (touchée, intouchable, saisie, insaisissable).

\section{Seuil Coma}

Picasso dessine dans l'espace une figure de minotaure ou de centaure [...], toute mentale, au moyen d'une torche électrique qui semble débobiner une ligne ininterrompue de lumière que l'appareil, réglé en longue pause, enregistre. Picasso ne voit presque pas ce qu'il a fait, il joue avec une lampe d'Aladin d'où surgirait, en un tournemain, une créature immatérielle qui n'est pas vraiment le produit d'un contour (ou d'un détour) mais d'une trajectoire fluide, souple et aérienne qui structure et enveloppe à la fois. ${ }^{31}$

Cette citation de Richard Conte qui évoque la photographie de Gjon Mili, Picasso et la fée électricité (Vallauris 1949), ne vient ici renforcer que cette idée que l'artiste est dans son expérience, que cela fait corps sans que nécessairement cela se trace sur un support. Certes, il y a ici un enregistrement photographique qui sera donné à l'artiste ultérieurement. Le centaure est dans la tête de l'artiste, c'est elle qui dirige le geste, qui sait à peu près ce que Picasso veut et va faire. L'amplitude du corps joue dans l'espace comme il peut jouer face à un papier, mais il ne voit pas ce qu'il a commencé à dessiner, cela ne reste visible, en quelque sorte, que dans les traces mémorielles. Cet exemple pour signifier que, pour moi, le coma, que je persiste à dire poreux, invente un espace qui est celui d'un corps réceptif non pas à tout mais à de fragiles traces (mécaniques, respiratoires, de noise) et que c'est avec cela qu'il faut, plus tard, sans le savoir au départ, composer, travailler, exercer). C'est pour cela que j'utilise le terme de Raum comme un embrassement. Le corps et/ou l'esprit d'un corps qui ne déambule plus dans un espace réinventent un espace beaucoup plus profond, plus loin parce qu'il charrie dans le même temps l'effet de perception et l'appréhension de l'infime mouvement des surfaces et des effets. Ce corps est un seuil qui accueille et repousse dans le même temps. Il finira par être s'œil. 
90 Le coma est un levier non pas pour un après mais pour toujours repenser toute l'absence de ce qui s'absente.

\section{Et dire encore comment ça se répète}

91 Avant le coma j'avais une vie qui s'est déposée dans la suivante. Ce « dépôt " pointe toujours le seuil limite du coma comme un nécessaire retour. L'activité, que certains nomment mon activisme (effréné), est évidemment liée à ce qu'on peut appeler une érotisation de la souffrance. Non pas jouir de la souffrance mais rejouer avec le corps (performance), avec la peinture (expérience des limites), avec la poésie (plongée dans un inconnu). Rejouer comme une sorte de mur où il faut revenir pour mourir et ne pas mourir. De le formuler ainsi met à mal ce qu'on attend habituellement d'une distance dans l'art, à faire l'art. Je ne crois pas que cette distance soit abolie, simplement il me semble qu'il s'agit de redonner du sens aux gestes de l'art, de redonner une sorte (forme) de resymbolisation à (de) l'œuvre, et mon expérience a précisément fortifié cela. Nous sommes dans une période où cette question est repensée par bon nombre d'artistes et de philosophes. Une sorte de manière de quitter ce que j'appellerais une sécheresse. Les lambeaux d'un corps, les brisures, les toiles qui revendiquent aussi d'être l'empreinte pas seulement de l'idée et de l'idéalité sont encore la conséquence d'une nouvelle habitation. Le coma fut un seuil, en tous les cas je le pense comme tel, qu'il ne s'agit surtout pas de mythifier, mais qui rappelle qu'on doit, comme artiste, se tenir à la brisée des événements, à la portée des formes de l'art le plus actuel et dans le même temps se saisir de ce que l'histoire nous permet d'être dans une plus totale complétude avec nous-mêmes et les autres.

Cette chose que j'appelle conscience témoigne d'une altérité avec le public, et cela permet, non pas de fonder une œuvre qui soit impérative, encore moins une œuvre avec laquelle on peut faire ce que l'on veut, cela permet d'ouvrir et de renouveler la part qui nous sépare et qui nous montre que nous sommes dans un parfait rapport d'altérité. 


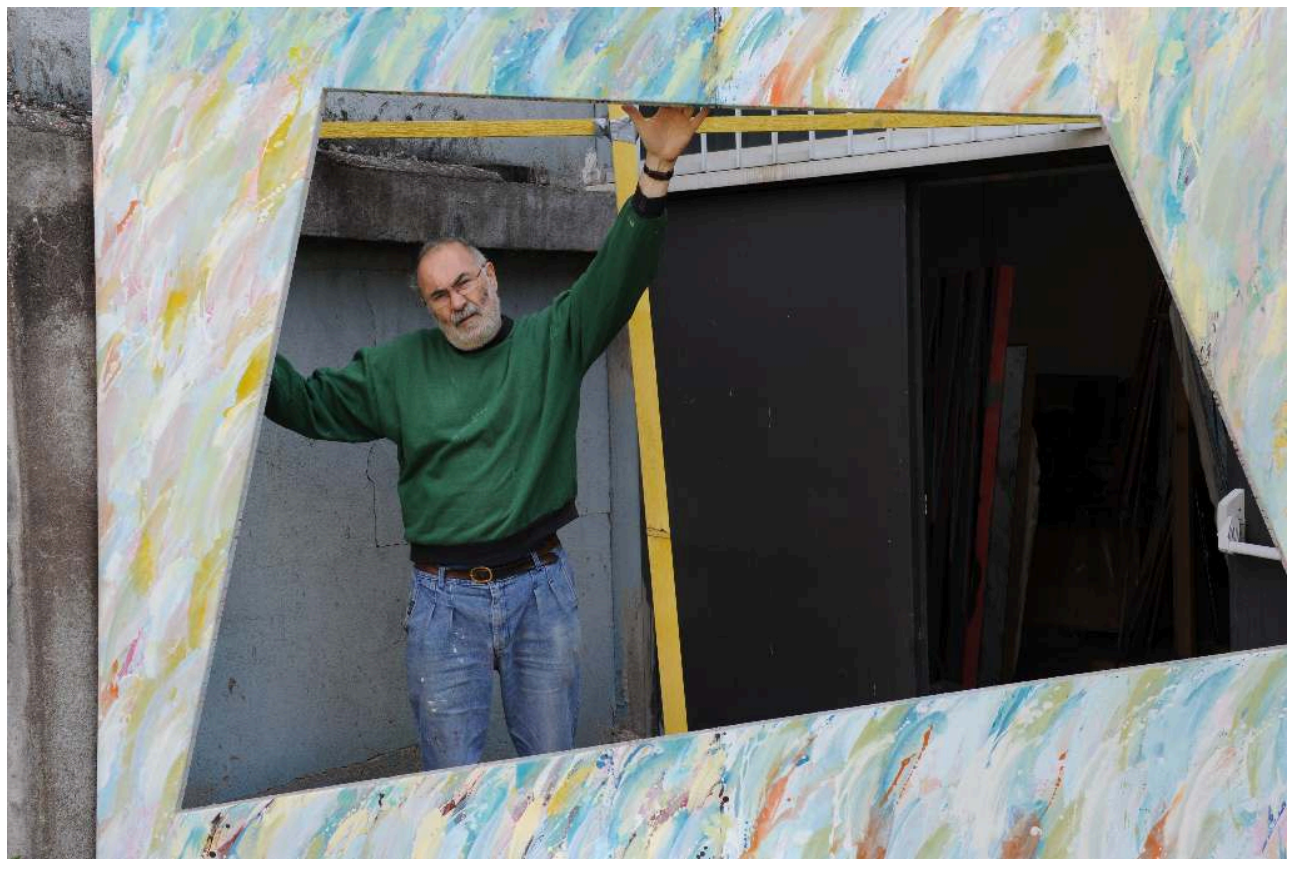

Photo P. Bouvier

\section{BIBLIOGRAPHIE}

Antoine Jean-Philippe, « Joseph Beuys : montrer ses blessures ou panser ses limites », Villa Gillet, cahier spécial, « Maladie et images de la maladie : 1790-1990 », Éditions Circé et Villa Gillet, 1995. Beckett Samuel, L'innommable, Éditions de Minuit, 2004 [1953].

Benjamin Walter, Chronique berlinoise, in Écrits autobiographiques, Christian Bourgois éditeur, coll. « Détroit », 1990.

Conte Richard, " photo-graphie », in Le dessin hors papier, Richard Conte (dir.), cérap université Paris 1, collection « Arts et monde contemporain », publication de la Sorbonne, 2009.

Delavaux Céline, L'art brut, un fantasme de peintre, Paris, éditions Palette, 2010.

Dorner Helmut, catalogue Han, Hans Thoma Museum in Bernau im Schwartzwald, 2001.

Lamarche-Vadel Bernard, Sanejouand, Jean-Michel, « Entretien mai-août 1986 », Octobre des arts, 1986.

L'Ollave Préoccupations, « Cécité passagère ", n 30, Jean de Breyne éditeur, Rustrel, 2008.

Payot Daniel, L'objet-fibule : Les petites attaches de l'art contemporain, L'Harmattan, collection «Esthétiques », Paris. 


\section{NOTES}

1. Walter Benjamin, Chronique berlinoise, in Écrits autobiographiques, Christian Bourgois éditeur, coll. « Détroit », 1990, p. 280.

2. Il faudra aussi évoquer la part théorique de mon travail dont on peut penser qu'il cherche à comprendre tant la dimension poïétique que d'altérite avec les œuvres de l'histoire.

3. Ce que je nomme l'enfance de l'art c'est ce moment où créer se fait dans une sorte de nécessité qui découvre ce qu'elle est, qui invente ses outils et ses concepts, qui reçoit des enseignements de toute nature qu'il faut partager, discuter, comprendre. L'opposition viendra plus tard lorsque la conscience de ce qui s'élabore trouve quelques justifications, quelques réponses.

4. On pourrait dire de route, ou de voiture. De route comme une déroute du déroulement normal d'une vie, de voiture comme d'un lieu dans lequel nous sommes immobiles et nous déplaçant. Ces notions, ces aperceptions furent au cours de toutes ces années très importantes.

5. Sur une surface, papier, drap récupéré, toile, bois. Déjà toutes sortes de surfaces.

6. L'écriture à l'opposé avait une forme d'antécédence. Mais j'y reviendrai.

7. Helmut Dorner, catalogue Han, Hans Thoma Museum in Bernau im Schwartzwald, 2001, p. 13.

8. Traduction Alexandra Pignol et Germain Rœsz.

9. Ici il faudrait dire que Germain Rœsz employé à la coopérative des bouchers et à l'abattoir de Colmar est une rencontre fortuite, un accident de la vie - avant l'autre autrement plus fondateur -, qui montre aussi une voie de couleur. Il me semble que le choix de faire une peinture plutôt peu épaisse montre cette couche ultime de la peau qui tremble, qui est habitée par tout ce qui la compose, par une multiplicité de couches (chez moi très liquides, comme une fluidité du penser), et encore pendant tout mon travail au recours à la toile libre comme une peau, qui se suspend justement comme une peau, comme je l'ai vu faire dans cet abattoir où je travaillais, mais aussi comme un corps aux esses accroché et toujours écartelé. La figure des bœufs dans l'abattoir n'est jamais très éloignée de la crucifixion, entre ouvrir et donner, montrer et éviscérer, écarteler et projeter.

10. Souvent des compétitions spontanées se réalisaient. J'ai ainsi pu voir un des ouvriers qui s'appelait René porter cinq sacs de sel (chacun pesant $50 \mathrm{~kg}$ ) en descendant des escaliers.

11. La perception, la réception d'un tableau est aussi de l'ordre de l'espace.

12. Dans l'atelier de Camille Hirtz.

13. J'ai pu dire plus tard qu'il s'agissait de l'apparition d'une œuvre de Michel Parmentier. La culture que je me fabriquerai viendra évidemment conforter ces découvertes initiales, brutales.

14. Il y en aura d'autres.

15. Je garde toujours pour cette dénomination une affection littéraire.

16. Les performances de poésie-action que je réalise se fondent sur la voix, le cri, le murmure s'originent peut-être dans la posture du muet.

17. Je peux dire aujourd'hui qu'il s'agit aussi d'une architecture qui dit dans le même temps sa peau, son épaisseur, sa déambulation possible et impossible, ses recoins, son sol, son ciel.

18. Samuel Beckett, L'innommable, Éditions de Minuit, 2004, [1953], p. 7.

19. Ibid., p. 11-12.

20. Nourri au sérum.

21. Il ne s'agissait pas véritablement de percer au sens de réussir mais bien de donner un autre terrain pour les rencontres humaines, une sorte de révolution à la mesure de nos moyens.

22. Céline Delavaux, L'art brut, un fantasme de peintre, Paris, éditions Palette, 2010.

23. Où dé-marquer.

24. Jean-Philippe Antoine, «Joseph Beuys : montrer ses blessures ou panser ses limites ", Villa Gillet, cahier spécial, « Maladie et images de la maladie : 1790-1990 », Éditions Circé et Villa Gillet, 1995, p. 36.

25. Presque au sens d'une reproduction. 
26. Anne Petrequin, enseignante-artiste me fait parvenir ce questionnaire (en juin 2005) par l'intermédiaire de Jean de Breyne. Expérience de cécité passagère-Trouble optique.

Si vous avez vécu des instants (fugitifs ou prolongés, légers ou intenses) d'aveuglement, de cécité passagère, d'éblouissement au cours de votre travail de création - avant, pendant ou après pourriez-vous relater cette expérience?

[Dans quelles circonstances?

Quelle durée? Quelle fréquence?

Qu'en dire ? Expérience agréable ou désagréable, subie ou attendue?

Quelles en seraient les causes, et les conséquences? etc.]

Il ne s'agit pas d'un questionnaire scientifique (ou «mystique ») mais d'une expérience à décrire, comme bon vous semble. Quelques mots, un texte, un schéma...

Ceci est envoyé à des écrivains, des artistes, des cinéastes, des créateurs, des collectionneurs...

Accepteriez-vous que votre réponse figure éventuellement dans une thèse d'arts plastiques ou dans un article? Merci de le confirmer.

Précisez votre fonction ou profession.

Pour me présenter: Titre de la recherche précédente (DEA cultures et civilisations du monde occidental / arts plastiques, 2001) :

« L'œil, l'antre et le crâne. Regard, suspens et vanité. D'une pratique de gravure »

Articles et chapitres d'ouvrage :

- "Réflexions sur le cuivre à graver ", dans Matière à symbolisation. Art, création et psychanalyse, sous la direction de Bernard Chouvier, textes de Didier Anzieu, Murielle Gagnebin, Jean-Michel Maulpoix et al., Lausanne, Delachaux et Niestlé, 2000.

- «Du Lièvre au Crâne, graver ", dans Le Portrait en abyme, sous la direction de Jacquie Barrai, Lyon, Aléas, 2001.

- «Quand l'œil est dans la plaque », Art et Thérapie : L'œuvre du regard, nº 88/89, décembre 2004.

Merci par avance pour votre participation.

Anne Petrequin, enseignante-artiste, agrégée d'arts plastiques

27. Daniel Payot, L'objet-fibule: Les petites attaches de l'art contemporain, L'Harmattan, collection «Esthétiques », Paris.

28. Bernard Lamarche-Vadel, Jean-Michel Sanejouand, «Entretien mai-août 1986 », Octobre des arts, 1986, p. 10-26.

29. Texte rédigé entre juin et septembre 2005, publié dans la revue L'Ollave Préoccupations, $\mathrm{n}^{\circ} 30$, p. 16-19, 2008.

30. Il faudrait parler d'arkhé-présence.

31. Richard Conte, "photo-graphie", in Le dessin hors papier, Richard Conte (dir.), cérap université Paris 1, collection «Arts et monde contemporain », publication de la Sorbonne, 2009, p. 25.

\section{RÉSUMÉS}

S'interrogeant sur les conditions de la genèse de son œuvre, le peintre et poète Germain Rœsz se remémore le long coma à l'issue duquel il s'est mis à manier la couleur - et les mots. Il propose le concept de Raum pour désigner cet état paradoxal où l'on touche et sent sans son corps. Chaque nouvelle création renouvelle ce lâcher-prise inaugural, inversant l'idée commune selon laquelle 
l'œuvre trouverait ses sources dans la seule histoire, dans l'addition des expériences de l'artiste, puisque le comateux précisément ne sent pas, puisque l'aveugle justement ne voit pas.

INDEX

Mots-clés : corps, mémoire, discontinuité, trace, chair, Raum, perception, aveuglement 